\title{
Social Creativity: Exploiting the Power of Cultures of Participation
}

\author{
Gerhard Fischer \\ Center for Lifelong Learning \& Design (L3D) \\ University of Colorado - Campus Box 430 \\ Boulder, CO 80303 , \\ USA \\ gerhardecolorado.edu
}

\begin{abstract}
Most interesting, important, and pressing problems facing our societies in the 21 st century transcend the individual human mind. They require social creativity to explore, frame, solve, and assess their solutions. To bring social creativity alive, cultures of participation need to be fostered and supported with socio-technical environments in which all stakeholders are able to express themselves, combine different perspectives, and generate new understandings.

Social creativity and cultures of participation are facilitated by meta-design that allows stakeholders to act as designers, contributors, and decision makers in personally meaningful activities.

This paper defines conceptual frameworks and briefly describes different applications contexts in gaining a deeper understanding of the challenges how to exploit the power of cultures of participation to enhance social creativity.
\end{abstract}

\section{INTRODUCTION}

The industrial information economy [1] has been focused on creating finished goods such as complete software systems, movies, and information repositories. The emerging networked information economy is democratizing the design and evolution of rich collaboratively constructed information environments [2] by creating socio-technical environments $[3,4]$ that support active contributors instead of passive consumers [5]. Cultures of participation break down the barriers and distinctions between designers and users and open up opportunities for social creativity [6]. Our research efforts are grounded in the basic assumption that the major role for new media and new technologies is not to deliver predigested information to individuals, but to provide them with opportunities and resources for engaging in meaningful activities, for participating in social debates and discussions, for creating shared understanding among all stakeholders, and for framing and solving authentic problems.

The paper briefly explores conceptual frameworks and different application contexts (ACs) - all of them focused on complex design problems including:

- AC-1: Envisionment and Discovery Collaboratory a table-top computing environment to collaboratively explore complex design problems;

- AC-2: Courses-as-Seeds - an educational environment supporting students as active contributors;

- AC-3: CreativeIT Wiki - a living organizational memory supporting researchers integrating creativity and information technologies;
- AC-4: Google SketchUp + 3D Warehouse + Google Earth — a large scale effort with world-wide contributions to model the whole world in 3D;

- AC-5: Socio-Technical Environments for People with Cognitive Disabilities — complementing weak internal scripts with powerful external scripts in support of distributed cognition;

- AC-6: Energy Sustainability - a socio-technical environment to reduce energy consumption.

\section{ADDRESSING IMPORTANT PROBLEMS}

Social creativity and cultures of participation offer important and interesting possibilities to cope with major problems [7] our societies are facing today including:

- problems of a magnitude which individuals and even large teams cannot solve (examples AC-3 and AC-4: to share research in creativity and to model all buildings in the world in 3-D);

- problems of a systemic nature requiring the collaboration of many different minds from a variety of background (example AC-1: urban planning problems as addressed by the Envisionment and Discovery Collaboratory);

- problems being poorly understood and ill-defined and therefore requiring the involvement of the owners of problems because they cannot be delegated to others (example AC-5: the unique needs of people with disabilities);

- problems modelling changing and unique worlds being dependent on open, living information repositories and tools (example AC-2: learners are not only consumers but also active contributors);

- problems requiring a change in human behaviour (example AC-6: energy sustainability).

Cultures of participation are facilitated and supported by a variety of different technological environments (such as: the participatory Web ("Web 2.0"), table-top computing, domain-oriented design environments); all of them contributing in different ways to the aims of engaging diverse audiences, enhancing creativity, sharing information, and fostering the collaboration among users acting as active contributors and designers. They democratize design and innovation [2] by shifting power and control towards users, supporting them to act as both designers and consumers ("prosumers") [8] and allowing systems to be shaped through real-time use. 


\section{SOCIAL CREATIVITY}

Social creativity explores computer media and technologies to help people work together. It is relevant to design because collaboration plays an increasingly significant role in design projects that require expertise in a wide range of domains. Software design projects, for example, typically involve designers, programmers, humancomputer interaction specialists, marketing people, and enduser participants [9]. Information technologies have reached a level of sophistication, maturity, cost-effectiveness, and distribution such that they are not restricted only to enhancing productivity but they open up new creative possibilities [10].

However, in a world in which solutions are neither given nor confined in one single mind [11], we need not only new models of collaboration, but also effective creativity support tools [12]. Social creativity requires active contributorspeople acting as designers in personally meaningful activities - not just consumers [5]. The necessity of involving and empowering users and allowing them to act as designers requires the expansion of the creative process from the individual to the group [10]. The sharing of products of individual creativity enables other people to work on them as a continuous activity without repeating unnecessary work. For example: the open-source movement $[13,14]$ demonstrates that the sharing of source code makes it possible for others to go forward when the original developers stop for various reasons, such as loss of interest or lack of time or new ideas.

\section{CULTURES OF PARTICIPATION}

The rationale and inspiration for cultures of participation comes from many sources, including the following prescriptive objectives and empirical observations:

- "The experience of having participated in a problem makes a difference to those who are affected by the solution. People are more likely to like a solution if they have been involved in its generation; even though it might not make sense otherwise" [15].

- "I believe passionately in the idea that people should design buildings for themselves. In other words, not only that they should be involved in the buildings that are for them but that they should actually help design them" [16].

- "Users that innovate can develop exactly what they want, rather than relying on manufacturers to act as their (often very imperfect) agents" [2].

- "The networked environment makes possible a new modality of organizing production: radically decentralized, collaborative, and non-proprietary" [1].

- "The opportunity to generate vibrant customer ecosystems where users help advance, implement, and even market new product features represents a largely untapped frontier for farsighted companies to exploit" [8].

\section{Social Creativity AND CUltures of Participation}

Where do new ideas come from in cultures of participation? The creativity potential is grounded in (1) user-driven innovations supported by meta-design environments, (2) taking advantage of breakdowns as sources for creativity, and (3) exploiting the symmetry of ignorance (meaning that all stakeholders are knowledgeable in some domains and ignorant in others) [17]. To increase social creativity requires: (1) diversity (each participant should have some unique information or perspective); (2) independence (participants' opinions are not determined by the opinions of those around them); (3) decentralization (participants are able to specialize and draw on local knowledge); and (4) aggregation (mechanisms exist for turning individual contributions into collections, and private judgments into collective decisions). In addition, participants must be able to express themselves (requiring technical knowledge how to contribute), must be willing to contribute (motivation), and must be allowed to have their voices heard (control).

\section{COMPONENTS OF A CONCEPTUAL FRAMEWORK}

\section{A. The Need for Multiple Voices: Exploiting Diversity and Distances}

In extended and distributed design projects, specialists from many different domains must coordinate their efforts despite large separations of time and distance. In such projects, collaboration is crucial for success, yet it is difficult to achieve. Complexity arises from the need to synthesize different perspectives, exploit conceptual collisions between concepts and ideas coming from different disciplines, manage large amounts of information potentially relevant to a design task, and understand the design decisions that have determined the long-term evolution of a designed artifact.

Social creativity and cultures of design thrive on the diversity of perspectives included by making all voices heard. It requires constructive dialogs between individuals negotiating their differences while creating their shared voice and vision. In our research, we have identified multiple voices by exploiting four different distances [6]:

- Voices from Different Places: Spatial Distance. Bringing spatially distributed people together with the support of computer-mediated communication allows the prominent defining feature of a group of people interacting with each other to become shared concerns rather than shared location. It further allows more people to be included, thus exploiting local knowledge.

- Voices from the Past: Temporal Distance. Design processes often take place over many years, with initial design followed by extended periods of evolution and redesign. In this sense, design artifacts (including systems that support design tasks, such as reuse environments [18]) are not designed once and for all, but instead evolve over long periods of time.

- Voices from Different Communities: Conceptual Distances. Design communities are social structures that enable groups of people to share knowledge and resources in support of collaborative design. Different communities grow around different types of design practice: (1) communities of practice (CoPs) [19] consist of practitioners who work as a community in a certain domain undertaking similar work; (2) communities of interest (CoIs) [20] bring together 
stakeholders from different CoPs to solve a particular (design) problem of common concern.

- Voices from Virtual Stakeholders: Technological Distances. Voices from virtual stakeholders can be embedded in computational artifacts. Designers use materials to construct design situations, and then listen to the "back-talk of the situation" they have created [21]. Computational design materials are able to interpret the work of designers and actively talk back to them.

\section{B. Meta-Design}

In past decades, design methodologies for most software systems have been focused to achieve better productivity and usability, and software design and human-computer interface $(\mathrm{HCI})$ research have made considerable progress for these objectives. However, we have entered a new phase of system development in exploring new application areas with a focus on collaborative design and social creativity by transcending existing boundaries and redistributing control among stakeholders [10]. In these developments people are not only using systems but they also are becoming involved in creating content and software to varying degrees.

Existing design methodologies are insufficient to cope with the emergence of situated and unintended requirements $[22,23]$. Socio-technical environments for which the design does not end at the time of deployment and whose success hinges on continued user participation [24] are needed.

Meta-design [25] is an emerging conceptual framework aimed at defining and creating socio-technical environments as living entities. It extends existing design methodologies focused on the development of a system at design time by allowing users to become co-designers at use time. Metadesign is grounded in the basic assumption that future uses and problems cannot be completely anticipated at design time, when a system is developed. Users, at use time, will discover mismatches between their needs and the support that an existing system can provide for them. Meta-design extends boundaries by supporting users as active contributors who can transcend the functionality and content of existing systems in personally meaningful activities. By facilitating these possibilities, control is distributed among all stakeholders in the design process.

\section{BRIEF DESCRIPTIONS OF OUR APPLICATION CONTEXTS}

This section describes a number of different application contexts $(A C s)$ in which we have explored different aspects of social creativity and cultures of participation.

\section{AC-1: Envisionment and Discovery Collaboratory (EDC)}

The EDC [17] is a long-term research platform exploring conceptual frameworks for collaborative design and social creativity in the context of complex design problems. It brings together participants from various backgrounds to frame and solve ill-defined, open-ended design problems. The knowledge to understand, frame, and solve these problems does not already exist [26], but is constructed and evolves during the solution process - an ideal environment to study social creativity. The EDC represents a sociotechnical environment incorporating a number of technologies, including table-top computing, the integration of physical and computational components supporting new interaction techniques, and an open architecture supporting meta-design activities. The vision of the EDC is to provide contextualized support for reflection-in-action [21] within collaborative design activities.

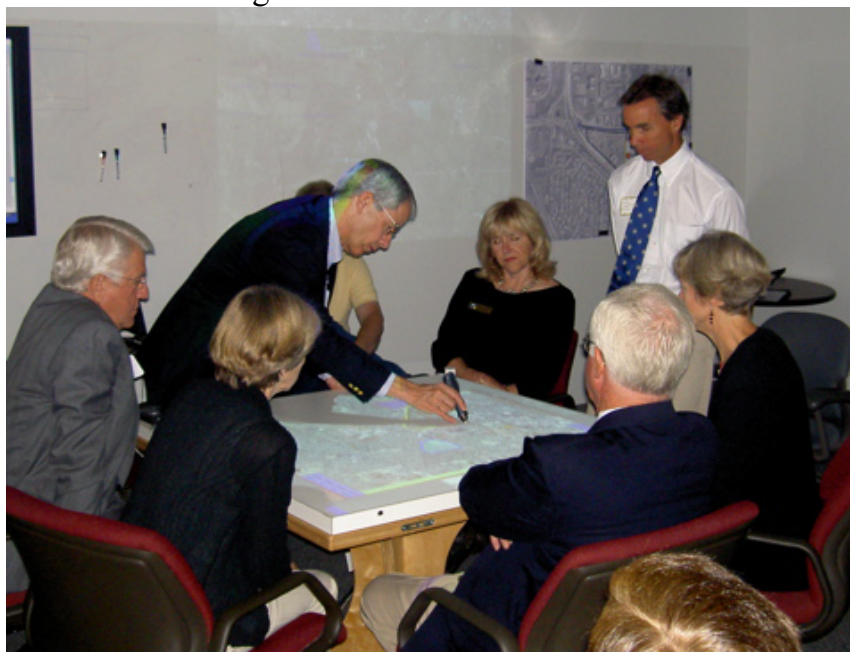

Figure 1: A community of interest using the EDC for a design session.

Figure 1 shows a design session between members of the Regents of the University of Colorado and the City Council of Boulder. The EDC supports face-to-face problem-solving activities by allowing the participants to discuss and explore problems while taking advantage of a shared construction space facilitated by a table-top computing environment. As participants interact with physical objects that are used to represent the situation currently being discussed and create design situation by sketching, corresponding computational representations are created and incrementally updated by using technologies that recognize these actions. Computergenerated information is projected back onto the horizontal physical construction area, creating an augmented reality environment. This physical construction is coupled with information relevant to the problem currently being discussed.

Grounded in a meta-design perspective, we have (1) included mechanisms within the EDC to allow participants to inject content into the simulations and adapt the environment to new scenarios; and (2) created ways to link to existing data and tools so that participants can draw on information from their own areas of expertise to contribute to the emerging, shared model. By exploring and supporting these activities, the EDC has given us insights into collaborative design that draw on both individual and social aspects of creativity.

\section{AC-2: Courses-as-Seeds}

Courses-as-seeds [27] is an educational model that explores meta-design and social creativity in the context of fundamentally changing the nature of courses taught in universities. Its goal is to create a culture of informed participation [28] that is situated in the context of university courses transcending the temporal boundaries of semesterbased classes. These courses are using wikis as course information environments (for examples see: http://13d.cs.colorado.edu/ gerhard /courses). Traditionally, the content of a course is defined by the resources provided by instructors (such as lectures, readings, and assignments), 
but, in courses-as-seeds, the instructor provides the initial seed rather than a finished product. By involving students as active contributors, courses do not have to rely only on the intellectual capital provided by the instructors but they are enriched on an ongoing basis by the contribution of all participants.

Courses-as-seeds represent a community-of-learners model [29] and explores new middle ground between adultrun and children-run education. All participants are active and the more skilled partners (experienced teachers and coaches) can provide leadership and guidance. The learners have opportunities to become responsible and organize their own learning, exploit their previous interests, and sustain their motivation to learn by having some control over their contributions.

\section{E. AC-3: CreativeIT Wiki}

Conventional wikis [8] have proven to be usable and useful to support communities, but one of their main limitations is their lack of support for different media types as they are applied to research in Creativity and IT as explored and supported by the NSF program CreativeIT (http://www.nsf.gov/pubs/2007/nsf07562/nsf07562.pdf). A consequence of this limitation is that communities (particularly those not focused on text) have only limited means to describe the research contributions. In our NSF supported research project (http://swiki.cs.colorado.edu/NGW) we are exploring factors in understanding and designing new wikis that can be used to support collaborative design and social creativity:

- Wikis have always had the goal of being open, simple, and low-threshold environments; this creates the challenge of increasing the expressiveness (the "high ceiling") required for creative activities in a wiki while retaining the low threshold;

- Most wikis have been used as content management systems in which individual contributions are accumulated; this raises the demand to improve support for dialogue, interpretation, and interactions;

- Many wikis lack participation of the communities; they are "systems built but users never come"; this requires a good seed, mechanism for evolutionary growth, and social reward structures to reach a "tipping point" [30] such that participation by the communities takes off.

These requirements, implementations, and assessments are currently analyzed in the CreativeIT Wiki (http://swiki.cs.colorado.edu/CreativeIT, see Figure 2), a socio-technical environment supporting the research community participating in the NSF CreativeIT program.

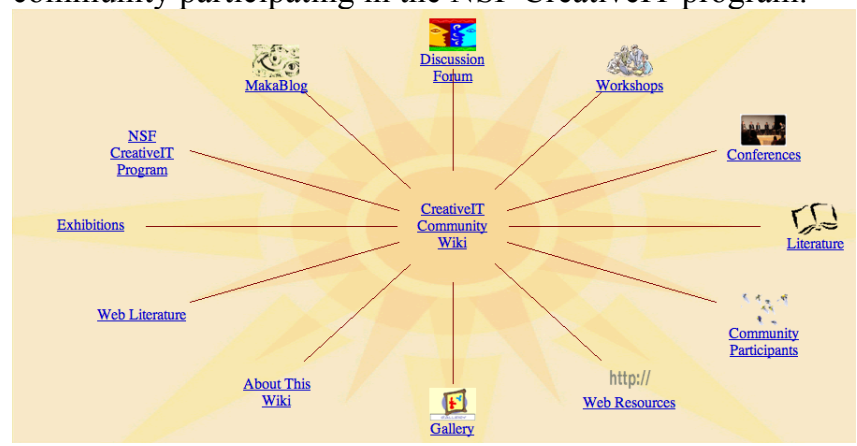

Figure 2: The CreativeIT Wiki

\section{F. AC-4: Google SketchUp + 3D Warehouse + Google Earth}

A research team at Google is working on the objective of having the whole world modeled in $3 \mathrm{D}$ and letting people use Google Earth to explore this virtual 3D world. This desirable objective cannot be achieved solely by a development team at Google due to the sheer amount of work it requires. The team at Google has chosen to create a socio-technical environment (supporting meta-design and wiki-style environments for sharing artifacts) by integrating SketchUp, 3D Warehouse, and Google Earth to support everyone motivated enough to participate in this effort. This project represents a unique, large-scale example in evaluating the conceptual framework for social creativity and cultures of participation.

SketchUp (http://sketchup.google.com/) is an interactive 3D modeling environment. Although SketchUp is a highfunctionality environment with a low threshold and a high ceiling, developing sophisticated models with SketchUp requires a nontrivial learning effort. In order to motivate enough people, powerful learning mechanisms for SketchUp are critical to allow everyone who wants to contribute to learn how to do so.

The 3D Warehouse (http://sketchup.google.com /3dwarehouse/) is an information repository for the collection of models created by any users who are willing to share the models they created through SketchUp. The 3D Warehouse contains hundred thousands of models from different domains, including buildings, houses, bridges, sculptures, cars, people, and pets. It supports collection mechanisms to organize models and supports ratings and reviews from community members.

Google Earth has the capability to show 3D objects consisting of users' submissions that were developed using SketchUp. Figure 3 shows downtown Denver modeled in 3D and displayed in Google Earth.

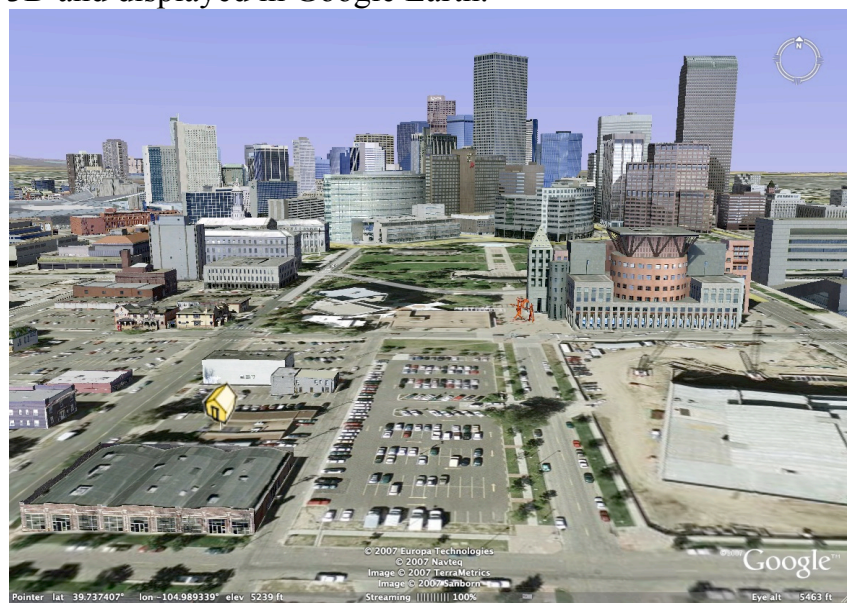

Figure 3: Downtown Denver modelled in Google Earth using SketchUp

\section{G. AC-5: Socio-Technical Environments for People with Cognitive Disabilities}

People with cognitive disabilities (“clients') represent a "universe of one" problem [31]: a solution for one person will rarely work for another. The "universe of one" conceptualization includes the empirical finding that (1) unexpected islands of abilities exist: clients can have unexpected skills and abilities that can be leveraged to 
ensure a better possibility of task accomplishment; and (2) unexpected deficits of abilities exist. Accessing and addressing these unexpected variations in skills and needs, particularly with respect to creating task support, requires an intimate knowledge of clients that only caregivers can provide. Currently, a substantial portion of assistive technology is abandoned after initial purchase and use causing the consequence that the very population that could most benefit from technology is paying for expensive devices that end up in the back of closets after a short time.

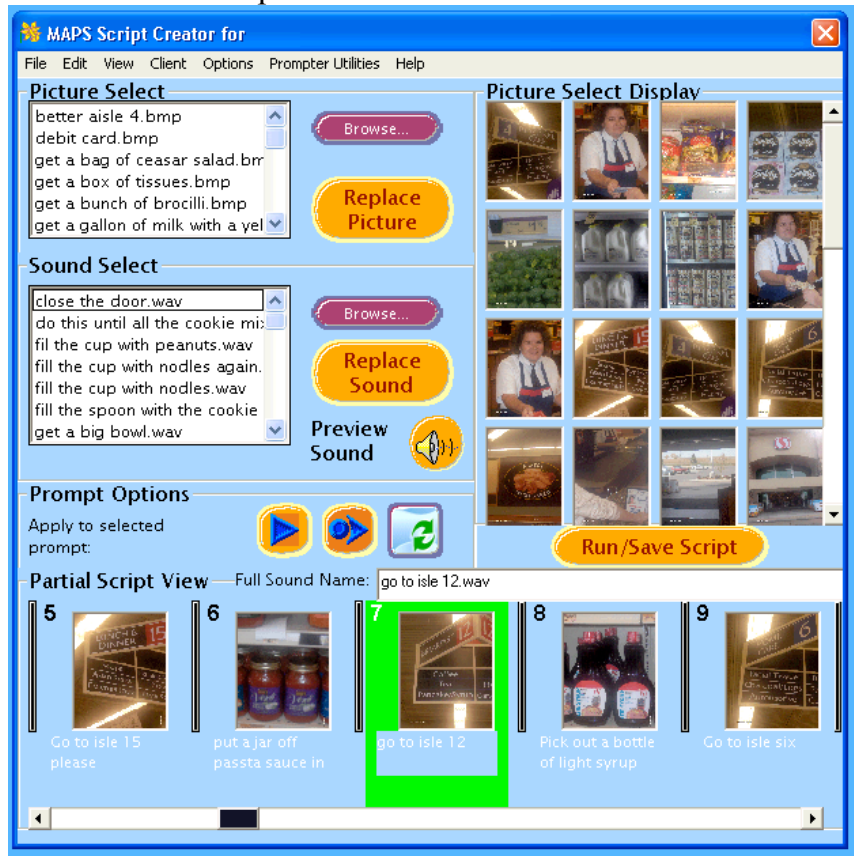

Figure 4: MAPS-DE—a Design Environment for Creating Scripts by Caregivers

In our long-term research project "CLever: Building Cognitive Levers to Help People Help Themselves" (http://13d.cs.colorado.edu/clever/) we have developed a specific socio-technical environment named Memory Aiding Prompting System (MAPS) allowing caregivers to create scripts that can be used by clients to support them in carrying out tasks that they would not be able to achieve by themselves. To account for the great diversity among clients, MAPS was developed as a meta-design environment, empowering caregivers to develop personalized prompting systems for the specific needs of individual clients. A unique challenge of meta-design in the domain of cognitive disabilities is that the clients themselves cannot act as designers, but the caregivers must accept this role.

$M A P S$ consists of two major subsystems (MAPS-DE, a design environment for caregivers and $M A P S-P R$, a prompting system for clients). MAPS-DE (see Figure 4) is implemented in a desktop environment for caregivers and employs web-based script and template repositories that allow content to be created and shared by caregivers of different abilities and experiences. MAPS-PR for clients (running on a wearable small computer) provides external scripts that reduce the cognitive demands of different activities for the clients

\section{H. AC-6: Energy Sustainability}

Energy sustainability is a theme of worldwide importance. Every aspect of our lives relies on energy, and societies as a whole are affected by the energy behaviour of its citizens. The development of a more responsible use of energy is one of the most important goals in our societies. The challenges of harvesting the benefits of technical innovations such as the Smart Grid (overlaying the electrical grid with a computational information system facilitating two-way communication) and advanced metering infrastructures (measuring, collecting, and analyzing energy usage by interacting with smart meter devices) are numerous: (1) most citizens are unaware of new technological developments; (2) information presentation is poorly designed; (3) the social context of individual energy use is ignored, and few interaction and collaboration mechanisms exist; and (4) feedback alone is not persuasive enough to change human behaviour. All of these challenges are grounded in the intersection of human behaviour (at the individual and social levels) and technology.

Our research efforts in this domain [32] are fostering and supporting cultures of participation (energy users acting as active decision makers) by exploiting the social creativity of designers from different domains to create meta-design environments providing support for visualizations, feedback, simulations, and sharing and analysing individual behaviour in communities. Figure 5 illustrates daily/weekly and realtime detailed feedback as follows: (1) the image (left side) shows a standard bill about energy consumption from a utility company obtained once a month (which most people find unintelligible); (2) the "Flower Pod" (right side, top) is an artistic illustration showing in a qualitative way the energy consumption in real time; and (3) the graph (right side, bottom) shows the competition between two dormitories at $\mathrm{CU}$ Boulder.

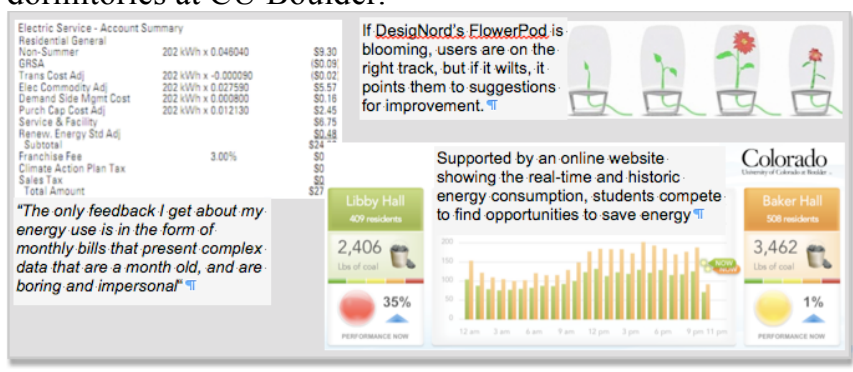

Figure 5: Empowering People to Reduce Energy Consumption

\section{LESSONS LEARNED FROM STUDYING THE DESIGN ACTIVITIES IN THE APPLICATIONS CONTEXTS}

For each of the ACs, we have conducted assessment studies grounded in the conceptual framework to identify the strengths and weaknesses and the lessons learned of our approach.

Assessment of the EDC. Our evaluations showed that EDC is an effective creativity support environment [33], empowering users in personally meaningful tasks to engage as active contributors, externalizing ideas and thereby allowing knowledge to be created, integrated, and disseminated. It has allowed users to interact and communicate with boundary objects supporting the generation of new ideas through the combination and improvement of existing ideas. Participants considered the sketching function allowing the creation of external representations to be crucial for the generation of objects "to-think-with" and "to-negotiate-about." 
In our assessment studies of the EDC, we have observed:

- More creative solutions to urban planning problems can emerge from the collective interactions with the environment by heterogeneous communities such as communities of interest rather than homogeneous communities such as communities of practice [20].

- Participants are more readily engaged if they perceive the design activities as personally meaningful by associating a purpose with their involvement [5].

- Participants must be able to naturally express what they want to say $[33,34]$.

- The interaction mechanisms must have a low threshold for easy participation and a high ceiling for expressing more sophisticated ideas [12].

- The representations of decisions and their consequences should be easily shared by other users for them to reflect upon others' perspectives and rationale [14].

- Visualizations of conflicting actions and decisions lead to lively discussion among participants and helps them reach a consensus or explore further alternatives [15].

Assessment of Course-as-Seeds. The courses-as-seeds model represents a system of values, attitudes and behaviours that differ radically from the traditional educational culture in which courses are conceived as finished products and students are viewed as consumers. Courses-as-seeds [27] creates a culture based on a designer mindset that emphasizes habits and tools that empower students to actively contribute to the design of their education (and eventually to the design of their lives and communities).

Assessment of the CreativeIT Wiki. Our findings can be summarized as follows: (1) current wiki-like environments are limited (there is a need to analyze and create additional objects such as mind maps, videos, anecdotes, and stories); (2) different modes of interacting with wikis need to be supported (including: face-to-face activities, synchronous, asynchronous); and (3) the right balance between supporting more complex interactions and more varied objects and avoiding the loss of the low threshold that wikis provide need to be found.

Assessment of SketchUp/3D Warehouse/GoogleEarth. In order for stakeholders to contribute 3D models, they need to learn SketchUp. This represents a major learning effort that needs to be facilitated by support environments. As the 3D Warehouse grows, support is not only needed for the contribution of additional 3D models, but the huge information environment needs to be organized and curatorial mechanisms need to be explored, designed, and implemented.

Assessment of MAPS. To assess the usability and usefulness of MAPS [31] equal attention was paid to the social environment and the performance environment in order to understand the world of the client and the process of adoption (or rejection) of MAPS. Understanding the process meant that the system had to be tested not in laboratories but with field trials in the real world by closely observing the participants in action and applying ethnographic participant observation as an analytical technique. Our assessment studies demonstrated the applicability of handheld multimedia prompting as task support for clients. One of the biggest indications of the success of MAPS was that clients/caregivers requested to keep the system after the studies were completed. MAPS empowered caregivers to create programs for individual users with cognitive disabilities. It represents an important example for democratizing design by supporting metadesign, embedding new technologies into socio-technical environments, and helping people with cognitive disabilities and their caregivers have more interesting and more rewarding lives.

Assessment of Energy Sustainability. Initial findings from our ongoing research efforts in this domain have shown that it is not enough to provide individuals with some feedback about their energy consumption (which they experience currently often as meaningless, dull, and complex). It is necessary to measure energy, but measurements by themselves are insufficient to change behaviour. Energy consumption needs to be visualized, socialized, and made understandable for consumers in order to motivate behavioural changes.

Summary. These assessment studies have emphasized our basic assumptions that social creativity and cultures of participation are enhanced by making all voices heard, harnessing diversity, and enabling people to be aware of and to access each other's work and ideas, relate them to their own, and contribute the results back to the community. While social creativity seen from this perspective is essential for framing and solving complex design problems, it contributes also to the invention and transformation of our social and cultural environments. With modern decentralization of knowledge into highly specialized niches, no single person is likely to have sufficient knowledge to solve a complex problem in any given field, and collaboration is therefore necessary. Our studies have provided evidence for our basic assumption that innovative socio-technical environments create feasibility spaces for new social practices.

\section{AN ENRICHED CONCEPTUAL FRAMEWORK}

Based on our original framework and new insights gained through its application in different contexts, we are able to articulate some initial success factors from our research including promises (new perspectives with potential that should be pursued) and pitfalls (problematic insights which should not be overlooked and misconceptions that must be exposed and examined). The most relevant factors are:

Create a deep understanding of the relationship between individual and social creativity. Our basic assumption is that social creativity and cultures of participation are necessities rather than luxuries for most interesting and important design problems in today's world. But there is ample evidence that there should be a "and" and not a "versus" relationship between individual and social creativity as aptly expressed by Rudyard Kipling "The strength of the pack is in the wolf, and the strength of the wolf is in the pack". This claim is strongly supported by other studies such as [11] and [35] and other conceptual frameworks such as the fish-scale model [36] which postulates that we should achieve "collective comprehensiveness through overlapping patterns of unique narrowness." Meta-design supports social creativity by 
democratizing design allowing all users to become participants in personally meaning problems.

Support Underdesign. An important objective of metadesign is to create important foundations for social creativity and cultures of participation by encouraging and supporting owners of problems to contribute user-generated content. Underdesign [37] allows owners of problems to adapt a system to local contingencies and conditions. It is a defining activity for meta-design aimed at creating design spaces for others. It assumes that the meaning, functionality, and content of a system are not fully defined by designers and user-representatives alone at design time, but are socially constructed throughout the entire design, deployment, and use cycles of the system.

Avoid group-think. Design communities are social structures that enable groups of people to share knowledge and resources. Different communities grow around different types of design practice; e.g., communities of practice [19] consisting of practitioners who work as a community in a certain domain undertaking similar work. As homogenous communities they can suffer from group think [38] by suppressing exposure to, and acceptance of, outside ideas.

Exploit the ecology of contributors. In cultures of participation there is no clear distinction between developers and users: all users are potential developers [20]. Being a consumer or being a designer is a not binary choice: it is rather a continuum ranging from passive consumer, to well-informed consumer, to end-user, to power user, to domain designer all the way to meta-designer [39].

Minimize the cost of contribution to encourage participation. People will decide on the worthiness of doing something by relating the (perceived) value of an activity to the (perceived) effort of doing it [25]. Experiences derived from our application contexts exposed two dimensions for achieving participation: individuals must perceive a value in contributing to an activity that is large enough to outweigh the effort [40]. Participation is a function depending on value and effort.

Value considerations can be greatly influenced by allowing people to engage in personally meaningful tasks, and it can induce them to serious working and learning. People are willing to spend considerable effort on things that are important to them, so the value dimension for truly personal meaningful activities is more important than the effort dimension.

The effort can be reduced (1) by lowering the threshold required to learn and make a contribution (e.g., by creating more effective learning systems for SketchUp); and (2) by taking advantage of derived information from the actions of participants.

Emphasize motivation. Without active contributions and participation from motivated users, social creativity and cultures of participation will not succeed. Although participants in community-based efforts (as exemplified by our application contexts) typically do not get paid for their contribution, there are other forms of external compensation contributing to motivation. Important motivational dimensions [35, 41] specifically supporting intrinsic motivation are: engagement in intellectually stimulating and personally enriching activities, generalized reciprocity, social recognition, rewards, and social capital.
Design and build social-technical environments. Social creativity and cultures of participation require the co-design of social and technical systems. They need to use models and concepts that focus not only on the artifact but exploit the social context in which the systems will be used [42]. Creativity flourishes best in a unique kind of social environment: one that is stable enough to allow continuity of effort, yet diverse and broad-minded enough to nourish creativity in all its subversive forms.

\section{CONCLUSIONS}

By studying social creativity in specific application contexts that foster and support cultures of participation, our research activities have contributed to and enriched conceptual framework. Achieving and supporting social creativity is not only a technical problem; it requires new cultures, new mindsets, and socio-technical environments that provide people with powerful media to express themselves and engage in personally meaningful activities. Research activities have only scratched the surface of exploiting the power of collective minds equipped with new media. The challenges of the complex problems that we all face make this approach not a luxury, but a necessity.

\section{ACKNOWLEDGEMENTS}

I thank the members of the Center for LifeLong Learning \& Design at the University of Colorado, who have made major contributions to the ideas and developments described in this paper. The research was supported in part by grants from the National Science Foundation, Google, and SAP.

\section{References}

[1] Y. Benkler, The Wealth of Networks: How Social Production Transforms Markets and Freedom. New Haven: Yale University Press, 2006.

[2] E. von Hippel, Democratizing Innovation. Cambridge, MA: MIT Press, 2005.

[3] E. Mumford, "Sociotechnical Systems Design: Evolving Theory and Practice," in Computers and Democracy, G. Bjerknes, et al., Eds., Aldershot, UK: Avebury, 1987, pp. 59-76

[4] G. Fischer and T. Hermann, "Socio-Technical Systems - A MetaDesign Perspective," International Journal for Sociotechnology and Knowledge Development (January-March 2011), vol. 3, pp. 133, 2011.

[5] G. Fischer. (2002). Beyond 'Couch Potatoes': From Consumers to Designers and Active Contributors, in FirstMonday (PeerReviewed Journal on the Internet). Available: http://firstmonday.org/issues/issue7_12/fischer/

[6] G. Fischer, et al., "Beyond Binary Choices: Integrating Individual and Social Creativity," International Journal of Human-Computer Studies (IJHCS) Special Issue on Computer Support for Creativity (E.A. Edmonds \& L. Candy, Eds.), vol. 63, pp. 482-512, 2005.

[7] G. Fischer, "Understanding, Fostering, and Supporting Cultures of Participation," ACM Interactions vol. XVIII.3 (May + June 2011), pp. 42-53, 2011.

[8] D. Tapscott and A. D. Williams, Wikinomics: How Mass Collaboration Changes Everything. New York, NY: Portofolio, Penguin Group, 2006.

[9] J. Greenbaum and M. Kyng, Eds., Design at Work: Cooperative Design of Computer Systems. Hillsdale, NJ: Lawrence Erlbaum Associates, Inc., 1991.

[10] National-Research-Council, Beyond Productivity: Information Technology, Innovation, and Creativity. Washington, DC: National Academy Press, 2003.

[11] W. Bennis and P. W. Biederman, Organizing Genius: The Secrets of Creative Collaboration. Cambridge, MA: Perseus Books, 1997. 
[12] B. Shneiderman, "Creativity Support Tools: Accelerating Discovery and Innovation," Communications of the ACM, vol. 50, pp. 20-32, 2007.

[13] E. S. Raymond and B. Young, The Cathedral and the Bazaar: Musings on Linux and Open Source by an Accidental Revolutionary. Sebastopol, CA: O'Reilly \& Associates, 2001.

[14] Y. Ye, et al., "The Co-Evolution of System and Community in Open Source Software Development," in Free/Open Source Software Development, S. Koch, Ed., Hershey, PA: Idea Group Publishing, 2004, pp. 59-82.

[15] H. Rittel, "Second-Generation Design Methods," in Developments in Design Methodology, N. Cross, Ed., New York: John Wiley \& Sons, 1984, pp. 317-327.

[16] C. Alexander, "The State of the Art in Design Methods," in Developments in Design Methodology, N. Cross, Ed., New York: John Wiley \& Sons, 1984, pp. 309-316.

[17] E. G. Arias, et al., "Transcending the Individual Human MindCreating Shared Understanding through Collaborative Design," ACM Transactions on Computer Human-Interaction, vol. 7, pp. 84-113, 2000

[18] Y. Ye and G. Fischer, "Reuse-Conducive Development Environments," International Journal Automated Software Engineering, Kluwer Academic Publishers, Dordrecht, Netherlands, vol. 12, pp. 199-235, 2005.

[19] E. Wenger, Communities of Practice - Learning, Meaning, and Identity. Cambridge, UK: Cambridge University Press, 1998.

[20] G. Fischer, "Communities of Interest: Learning through the Interaction of Multiple Knowledge Systems," in 24th Annual Information Systems Research Seminar In Scandinavia (IRIS'24), Ulvik, Norway, 2001, pp. 1-14.

[21] D. A. Schön, The Reflective Practitioner: How Professionals Think in Action. New York: Basic Books, 1983

[22] L. A. Suchman, Plans and Situated Actions. Cambridge, UK: Cambridge University Press, 1987.

[23] T. Winograd and F. Flores, Understanding Computers and Cognition: A New Foundation for Design. Norwood, NJ: Ablex Publishing Corporation, 1986.

[24] A. Henderson and M. Kyng, "There's No Place Like Home: Continuing Design in Use," in Design at Work: Cooperative Design of Computer Systems, J. Greenbaum and M. Kyng, Eds., Hillsdale, NJ: Lawrence Erlbaum Associates, Inc., 1991, pp. 219240.

[25] G. Fischer and E. Giaccardi, "Meta-Design: A Framework for the Future of End User Development," in End User Development, H. Lieberman, et al., Eds., Dordrecht, The Netherlands: Kluwer Academic Publishers, 2006, pp. 427-457.

[26] Y. Engeström, "Expansive Learning at Work: Toward an Activity Theoretical Reconceptualization," Journal of Education and Work, vol. 14, pp. 133-156, 2001.

[27] R. dePaula, et al., "Courses as Seeds: Expectations and Realities," in Proceedings of The European Conference on ComputerSupported Collaborative Learning, P. Dillenbourg, et al., Eds., Maastricht, Netherlands, 2001, pp. 494-501.
[28] G. Fischer and J. Ostwald, "Knowledge Communication In Design Communities," in Barriers and Biases in Computer-Mediated Knowledge Communication, R. Bromme, et al., Eds., New York, N.Y.: Springer, 2005, pp. 213-242.

[29] B. Rogoff, et al., "Models of Teaching and Learning: Participation in a Community of Learners," in The Handbook of Education and Human Development - New Models of Learning, Teaching and Schooling, D. R. Olsen and N. Torrance, Eds., Oxford: Blackwell, 1998, pp. 388-414.

[30] M. Gladwell, The Tipping Point: How Little Things can Make a Big Difference. New York, NY: Back Bay Books, 2000.

[31] S. P. Carmien and G. Fischer, "Design, Adoption, and Assessment of a Socio-Technical Environment Supporting Independence for Persons with Cognitive Disabilities," in Proceedings of CHI 2008, Florence, Italy: ACM, 2008, pp. 597 - 607.

[32] H. Dick, et al., "From Consumers to Owners: Using Meta-design Environments to Motivate Changes in Energy Consumption," in End-User Development (Third International Symposium, Torre Canne, Italy, June). vol. LNCS 6654, M. F. Costabile, et al., Eds., Heidelberg: Springer, 2011, pp. 319-324

[33] H. Eden, "Getting in on the (Inter)Action: Exploring Affordances for Collaborative Learning in a Context of Informed Participation," in Proceedings of the Computer Supported Collaborative Learning (CSCL '2002) Conference, G. Stahl, Ed., Boulder, CO, 2002, pp. 399-407.

[34] B. A. Myers, et al., "Invited Research Overview: End-User Programming," in Human Factors in Computing Systems, CHI'2006 (Montreal), 2006, pp. 75-80.

[35] M. Csikszentmihalyi, Creativity - Flow and the Psychology of Discovery and Invention. New York, NY: HarperCollins Publishers, 1996.

[36] D. T. Campbell, "Ethnocentrism of Disciplines and the Fish-Scale Model of Omniscience," in Interdisciplinary Collaboration - An Emerging Cognitive Science, S. J. Derry, et al., Eds., Mahwah, NJ Lawrence Erlbaum, 2005, pp. 3-21.

[37] S. Brand, How Buildings Learn: What Happens After They're Built. New York: Penguin Books, 1995.

[38] I. Janis, Victims of Groupthink. Boston: Houghton Mifflin, 1972.

[39] J. Preece and B. Shneiderman, "The Reader-to-Leader Framework: Motivating Technology-Mediated Social Participation," AIS Transactions on Human-Computer Interaction, vol. 1, pp. 13-32, 2009

[40] J. Grudin, "Groupware and Social Dynamics: Eight Challenges for Developers," Communications of the ACM, vol. 37, pp. 92-105, 1994.

[41] Y. Benkler and H. Nissenbaum, "Commons-based Peer Production and Virtue," Political Philosophy, vol. 14, pp. 394-419, 2006.

[42] H. Zhuge, "Semantic linking through spaces for cyber-physicalsocio intelligence: A methodology," Artificial Intelligence, vol. 175, pp. 988-1019, 2011. 